\title{
Avoid Certain Frustration-Or Maybe Not?
}

\author{
Neven Sesardić
}

In the situation known as the cable guy paradox, the expected utility principle and the avoid certain frustration (ACF) principle seem to give contradictory advice about what one should do. This article tries to resolve the paradox by presenting an example that weakens the grip of ACF: a modified version of the cable guy problem is introduced in which the choice dictated by ACF loses much of its intuitive appeal.

Keywords cable guy paradox; decision theory; expected utility; certain frustration; Alan Hájek

DOI:10.1002/tht3.390

In the situation known as the cable guy paradox (Hájek 2005), the expected utility principle and the avoid certain frustration (ACF) principle seem to give contradictory advice about what one should do. Although Alan Hájek, the father of the cable guy paradox, ultimately rejects the advice derived from ACF, he concedes that something of a paradox still remains.

This article tries to remove the lingering appearance of a paradox by presenting an example that weakens the grip of ACF: a modified version of the cable guy problem will be introduced in which the choice dictated by ACF loses much of its intuitive appeal.

Let us first recall the original paradox. The cable guy will come to your home tomorrow sometime after 8 a.m. and before 4 p.m. Your friend proposes a bet, on even money, on whether the cable guy will come in the morning (between 8 and 12) or in the afternoon (between 12 and 4). You can choose which option you want to bet on. Since the probability of MORNING or AFTERNOON is the same (one half) and since your potential gain and loss are also the same (the assumption is that nothing else matters but money), it seems you should be indifferent between the two options. This answer is based on the expected utility principle.

Then Hájek introduces an alternative argument (which he in the end rejects) in favor of AFTERNOON. If you bet on MORNING, there will inevitably be some moment in the morning (before the cable guy arrives) when you will be aware that some of "your" time has elapsed, and consequently the probability of AFTERNOON will at that moment be higher than the probability of MORNING. Since it is certain that at some point you will be frustrated with your choice if you choose MORNING, but it is not certain that you will be frustrated with your choice if you choose AFTERNOON, there is a suggestion that you 


\begin{tabular}{|c|c|c|c|}
\hline$\varepsilon_{1}$ & MORNING $-\varepsilon_{1}$ & $\varepsilon_{2}$ & AFTERNOON $-\varepsilon_{2}$ \\
\hline
\end{tabular}

Figure 1: The cable guy problem modified.

should bet on AFTERNOON. This suggestion is based on ACF, which Hájek formulates in the following way:

Avoid Certain Frustration (ACF) Principle: Suppose you now have a choice between two options. You should not choose one of these options if you are certain that a rational future self of yours will prefer that you had chosen the other one - unless both your options have this property. (Hájek 2005, p. 114)

Hájek notes that the choice to bet on MORNING "falls squarely under the purview" of ACF, which conflicts with what is recommended by the principle of expected utility (namely, that one should be indifferent between the two choices). One way to solve the paradox would be to put ACF itself in question by constructing a counterexample in which all of Hájek's conditions for applying ACF are satisfied but where there is no imperative to ACF. This is what I will attempt to do.

Let us first change the conditions of the bet. Let us divide the entire period between 8 a.m. and 4 p.m. into the following four temporally consecutive subintervals: $\varepsilon_{1}$ (the trillionth-of-a-second interval after 8 a.m.), MORNING $-\varepsilon_{1}$ (the rest of the morning), $\varepsilon_{2}$ (the trillionth-of-a-second interval after 12 noon), and AFTERNOON $-\varepsilon_{2}$ (the rest of the afternoon).

Or, to put it in more exact terms:

$$
\begin{aligned}
& \varepsilon_{1}=(8,8+\varepsilon] \\
& \text { MORNING }-\varepsilon_{1}=(8+\varepsilon, 12] \\
& \varepsilon_{2}=(12,12+\varepsilon] \\
& \text { AFTERNOON }-\varepsilon_{2}=(12+\varepsilon, 4)
\end{aligned}
$$

The division is shown in Figure 1 (not drawn to scale), which represents the whole period between 8 a.m. and 4 p.m.

Now in the new version of the cable guy problem, the choice is between betting on one of the two time periods in the figure that are represented by white and gray, respectively. Your options now are betting either on $W$ (the white segment) or on $G$ (the gray segment):

Betting on $W$ means you win if the cable guy arrives during $\varepsilon_{1}$ or during (AFTERNOON $-\varepsilon_{2}$ ), and you lose otherwise.

Betting on $G$ means you win if the cable guy arrives during (MORNING $-\varepsilon_{1}$ ) or during $\varepsilon_{2}$, and you lose otherwise.

The difference between this and the original cable guy scenario is that now your first option includes betting on a time that consists of two noncontiguous intervals: $\varepsilon_{1}$ and (AFTERNOON $-\varepsilon_{2}$ ). However, the intervals $W$ and $G$ are still of the same duration 
(exactly 4 hours each), so according to the expected utility principle you should again be indifferent between the two choices.

ACF, however, clearly favors $G$, because choosing $W$ would lead to certain frustration of your future rational self (during the initial interval $\varepsilon_{1}$ ). For, whether the cable guy arrives during $\varepsilon_{1}$ or not, there will certainly be an interval, during $\varepsilon_{1}$, when you will regret choosing $W$, since you will realize that some of "your" time has elapsed without the cable guy coming and that consequently your chance of winning just became (be it even very slightly) lower than .5 .

Although it is indeed certain that a rational future self of yours will regret the choice of $W$, let us look at how another rational future self of yours would feel at a still later time. If you choose $G$, what is the probability that a later self of yours will at some future time regret that choice? Obviously, this probability is equal to the probability that the cable guy does not arrive during the interval $(8,8+2 \varepsilon]$. Explanation: a future self will regret choosing $G$ just as long as the cable guy has not yet arrived and the remaining portion of $G$ time ahead is shorter than the remaining portion of $W$ time. If after 8 o'clock the short period of the duration $2 \varepsilon$ expires without the cable guy arriving, then a future self would regret choosing $G$.

Since $2 \varepsilon$ is an extremely small part of the whole time period within which the cable guy can arrive, the probability that the guy will not come during that period is very high. So the probability that a "post- $2 \varepsilon$ " rational future self of yours will be frustrated if you choose $G$ is very, very high (although lower than unity). And the probability that a rational later self of yours will regret making choice $G$ in this kind of scenario can be made as close to unity as we like - by making the interval $2 \varepsilon$ smaller and smaller.

Now back to ACF. ACF is based on the idea that, other things being equal, a judgment of one's later rational self (during $\varepsilon_{1}$ ) should carry more weight (because it is based on more comprehensive evidence) than the judgment of one's earlier rational self (before 8 a.m.). But why shouldn't we then extend this reasoning and also take into account a judgment of an even later rational self (after $\varepsilon_{1}$ ), especially since this self is also expected to be frustrated with a very high probability? Admittedly, choosing $W$ entails that one's early future rational self-during $\varepsilon_{1}$ - will certainly regret one's decision. Choosing $G$, however, entails that one's later future rational self will regret one's decision with the probability that can be made as close to unity as desired (by making $\varepsilon$ smaller and smaller). The probabilities of frustration in the cases of the two decisions can be made as close to one another as you like.

But then it is unclear why one should follow ACF. There is nothing obviously irrational about going against ACF and betting on $W$, with its certain frustration of one's earlier future self, rather than betting on $G$, with its frustration of one's later future self with a probability that can be made as close to certainty as we please.

Hájek was not convinced by my argument, though. This is his response (personal communication):

But in a way [your example] only strengthens my paradoxical reasoning. Suppose you bet on $G$. You will certainly be glad for a little while, during period $\varepsilon 1$. You will 
enjoy watching $W$ dwindle, for a short time. Then yes, very probably you will begin a period of frustration as we enter the gray region, and $G$ begins to dwindle. So you will experience:

(i) gladness certainly, and

(ii) frustration (only) very probably.

On the other hand, if you bet on $\mathrm{W}$, you will experience:

(i) frustration certainly, and

(ii) gladness (only) very probably.

So there are now two reasons to prefer $G$ to $W$ !

1. CERTAIN gladness is better than PROBABLE gladness.

2. PROBABLE frustration is better than CERTAIN frustration.

My argument based on ACF only gave you one reason to do so!

Note that Hájek stresses that he does not ultimately advocate ACF-indeed the conclusion of his paper was to reject it - nor the reasoning in the above passage. However, he offers both as prima facie plausible, and finds it surprising that they must be rejected.

I disagree with Hájek's response, but I think it is very useful because it helps us probe deeper into the core of the cable guy paradox.

His two reasons for preferring $G$ over $W$ are: on one hand, certain gladness (the result of choosing $G$ ) is better than merely probable gladness (the result of choosing $W$ ), and on the other hand, merely probable frustration (the result of choosing $G$ ) is better than certain frustration (the result of choosing $W$ ).

The reasoning would be convincing if certain and probable frustration - or certain and probable gladness - associated with the two respective decisions were quantitatively the same. But they are not.

Take frustration first. Recall that here we are talking about the frustration of one's future self if a part of one's time elapses without the cable guy arriving, which proportionately decreases one's chances of winning the bet. Now it is obvious that the (certain) frustration resulting from choosing $W$ will be very small. The worst case scenario would be that your future self would realize toward the end of the minuscule interval $\varepsilon_{1}$ that almost one trillionth of a second of "your" time has elapsed, and that consequently your chances of winning the bet would have diminished only by a practically negligible amount.

If you choose $G$, though, your future frustration will indeed not be certain but it will be practically indistinguishable from certainty. In addition, this frustration will not be contained within the very narrow range $\varepsilon$, as in the choice of $W$. On the contrary, choosing $G$ you will risk a much larger frustration, that is, realizing with regret that a much higher proportion of your time than $\varepsilon$ has elapsed without the cable guy coming. (At the limit there is a probability of around $1 / 2$ that you will go through successive and ever larger levels of frustration until almost your entire time expires without the cable guy arriving.) 
Briefly, if choosing between $W$ and $G$ comes down to choosing between the certainty of a virtually zero frustration and the high probability (very closely approaching certainty) of a much larger frustration, it is far from clear that choosing $G$ is preferable.

Ditto for gladness. Is Hájek right that certain gladness is better than merely probable gladness? Again, no. When a certain but tiny gladness is pitted against a much larger gladness that is virtually (though not actually) certain, an argument could be made for choosing the latter. Let me stress I do not want to defend that other argument. I merely use it to show that the reasoning in favor of ACF that Hájek finds prima facie plausible loses its force on closer inspection.

Contra Hájek, two out of three possible reasons favor choosing interval $W$ over interval $G$. First, in the case of choosing $W$ potential frustration would be smaller and potential gladness greater (than if one chose $G$ ). Second, deferring to the judgment of one's later rational self (in the spirit of Van Fraassen's reflection principle) also supports choosing $W$, because an almost certain frustration expected by one who chose $G$ would happen after a certain frustration expected by one who chose $W$. (And mutatis mutandis for gladness). Third, the only reason apparently favoring interval $G$ is that the future frustration is certain for one who chooses $W$, though not for one who chooses $G$. But, to repeat, the difference between certainty and a probability that we can make as close to certainty as we like should not carry much weight.

So should one always follow ACF? Perhaps not.

\section{Acknowledgments}

I would like to thank Nathan Cofnas, Rafael De Clercq, Alan Hájek, and Matej Sušnik for useful comments on earlier drafts of this paper.

\section{Reference}

Hájek, A. “The Cable Guy Paradox.” Analysis 65 (2005): 112-119. 